\title{
On the Relation between Iron Oxide in Slag and Oxygen in Steel in LD Converter*
}

\author{
By Shinji TSUDA, ${ }^{* *}$ Takami IKEDA, ${ }^{* *}$ and Katsukiyo MARUKAWA ${ }^{* *}$
}

\section{Synopsis}

The relation between iron oxide in slag and oxygen in metal in 160-t $L D$ converter are studied, and the main results obtained are as follows:

(1) During the period between end point and tapping the oxygen content in metal changes in a range of $\pm 150 \mathrm{ppm}$ in low carbon steel $(0.04$ to $0.08 \% \mathrm{C}$ ).

(2) This change of oxygen in metal depends on the total FeO content of slag. When the total $\mathrm{FeO}$ content in slag is high, oxygen content in metal increases between end point and tapping. On the other hand, when the total $\mathrm{FeO}$ content is low, the oxygen content decreases.

(3) At the end of oxygen blowing the slag temperature is higher than that of metal. The difference in temperatures seems to be over $50^{\circ} \mathrm{C}$. This difference, however, vanishes during the period between end point and tapping. This decrease of slag temperature after the end of blowing reduces the degree of oxidation of slag.

(4) As a consequence, the oxidizing potential of slag becomes lower than that of metal at tapping. This case occurs in approximately one third of the heats of low carbon steels. In medium and high carbon steels, however, this case scarcely occurs.

\section{Introduction}

For all the refining furnaces the slag control is one of the most important subjects in steelmaking. Oxygen in steel, which has a large influence on the steel quality, is closely connected with $\mathrm{FeO}$ in the slag.

$\mathrm{FeO}$ in slag in the oxygen converter, however, has a role different in the refining from other furnaces; that is, while $\mathrm{FeO}$ in slag before the blocking in B.O.H. (Basic Open Hearth furnace) has a large influence on the oxygen content in metal at same carbon content, $\mathrm{FeO}$ in the slag at the end point of B.O.F. (Basic Oxygen Furnace or LD Converter) is said to have no influence on oxygen converter. ${ }^{1), 2)}$

Experience shows, on the other hand, that high content of T.Fe (the total iron content of iron oxide) in slag can occasionally be the cause of weak deoxidation in actual operation. These cases prove that the T.Fe in slag is related to the oxygen content in metal.

It is by this reason that the present authors have studied the relation between the iron oxide content in slag and the oxygen content in metal at and after the end point of B.O.F. operation. The bomb method for the metal sampling, the Ar carrier arc fused infrared spectrophotometry method for the oxygen analysis, and the fluorescent X-ray spectrophotometry method for the slag analysis were used.

\section{Test Result}

1. On the Difference of the T.Fe in the B.O.H. and the B.O.F.

Figure 1 shows the data from 160-t B.O.F.'s as well

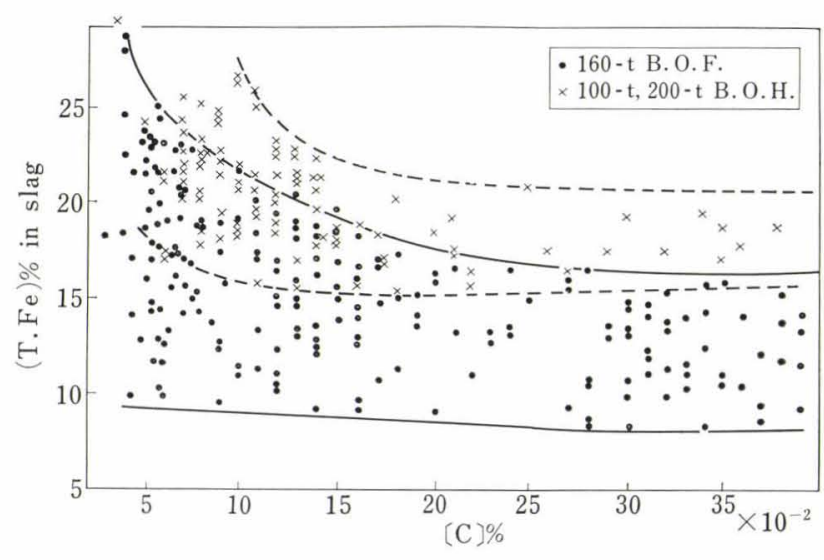

Fig. 1. Relation between $[\mathrm{C}] \%$ and (T.Fe) $\%$ at end point in converter and open hearth

as 100- and 200-t B.O.H.'s. In this figure, it is to be seen that there is a large difference in the $\mathrm{T} . \mathrm{Fe} \%$ in slag between those furnaces. That is, $\mathrm{T} . \mathrm{Fe} \%$ in B.O.F. is $70 \%$ of that in B.O.H., and the degree of scatter in B.O.F. is twice the number in B.O.H.

\section{On the Behavior of Oxygen after the End Point}

Metals and slags were sampled both at the end point and at the tapping. The sampling at the end point was done from several seconds to several minutes after the oxygen blow-down, and that at the tapping was from 3 to $5 \mathrm{~min}$ after the end point. Metal was sampled at the position of $30 \mathrm{~cm}$ below the slag-metal interface.

The relation of the oxygen content between at the end point and at the tapping is shown in Fig. 2. This figure shows that there is no large change of oxygen during the period from the end point to the tapping. However the degree of scatter of oxygen in low carbon range is rather large.

The nature of this scatter becomes clear in Fig. 3, which shows the change in the oxygen content in metal after the end point in terms of the $[\Delta \mathrm{O}][\Delta \mathrm{O}]=[\mathrm{O}\rceil$ at the tapping - $[\mathrm{O}]$ at the end point) vs. [O] at the end point. It will be noticed in Fig. 3 that the $[\Delta \mathrm{O}]$ of the medium and high carbon steels is small, whereas [ $\lrcorner \mathrm{O}]$ of low carbon steels is large, the range of this scatter being over $\pm 150 \mathrm{ppm}$. This result shows that a large change occurs in the oxygen in metal during some minutes of metal's stay in the furnace.

\section{On the Behavior of Carbon after the End Point}

The change of carbon content is shown in Figs. 4

* Originally published in Tetsu-to-Hagané, 56 (1970), 186 in Japanese. English version received October 1, 1969.

** Wakayama Steel Works, Sumitomo Metal Industries, Ltd., Minato, Wakayama 641. 


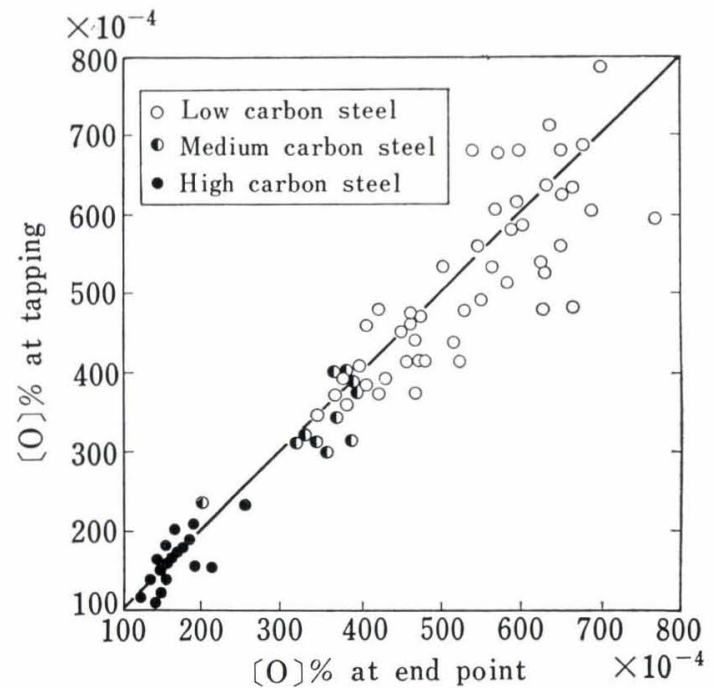

Fig. 2. Relation between oxygen content at end point and at tapping

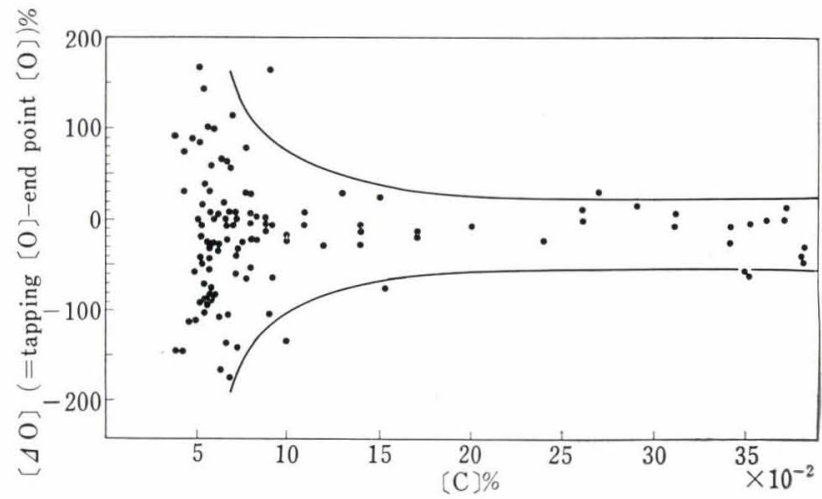

Fig. 3. Relation between change of oxygen after end point and carbon at end point in converter

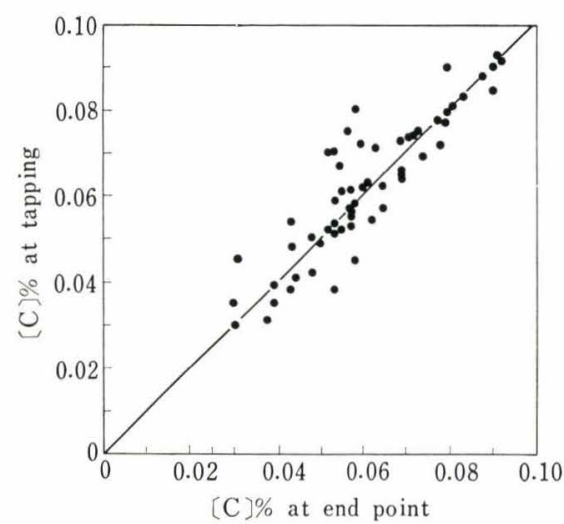

Fig. 4. Relation between carbon contents at end point and at tapping for low carbon steels

and 5. In Fig. 4, which is for low carbon steels, there is little change in the carbon content from the end point to the tapping, though the scatter of data is large; namely, the decarburization reaction in low carbon steels in the furnace is not intensive.

For medium and high carbon steels, on the other hand, the decrease of carbon content can be as much as $0.01 \%$ during several minutes of stay in the furnace,

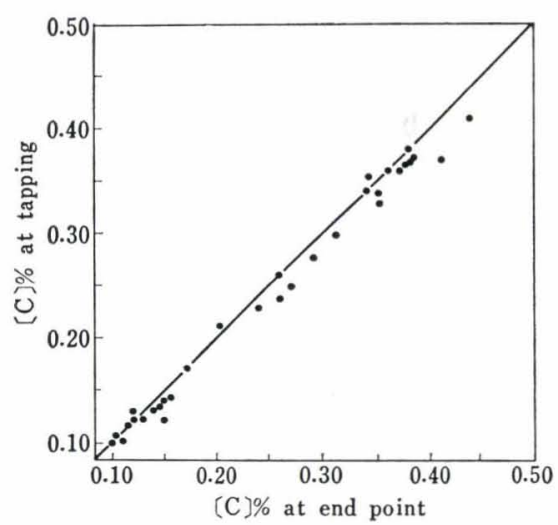

Fig. 5. Relation between carbon contents at end point and at tapping for medium and high carbon steels

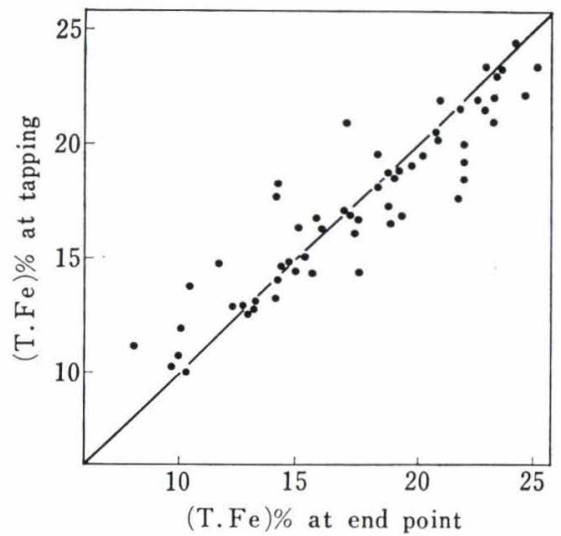

Fig. 6. Relation between T.Fe\% at end point and at tapping for low carbon steels

Fig. 5.

\section{On the Behavior of Slag T.Fe after the End Point}

The behavior of T.Fe at the low carbon steel is shown in Fig. 6. For heats with high T.Fe at the end point, the T.Fe decreases during the period from the end point to the tapping. On the other hand, for heats with low T.Fe at the end point, it increases during the same period.

The change of T.Fe for medium and high carbon steels is shown in Fig. 7 : the T.Fe decreases in general during the period from the end point to the tapping, especially so for high carbon steels.

\section{On the Difference in Temperatures of Slag and Metal after the End Point}

The change of slag temperature after the end point is shown in Fig. 8. A decrease of temperature from $30^{\circ}$ to $50^{\circ} \mathrm{C}$ occurs during the period from the end point to the tapping, and a difference exists in the temperature between slag and metal at the end point. It disappears, however, during those several minutes from the end point to the tapping.

The temperature difference between slag and metal at the end point was investigated for many heats. The result is shown in Fig. 9. The heats tested included low carbon, medium carbon, and high carbon steels. 


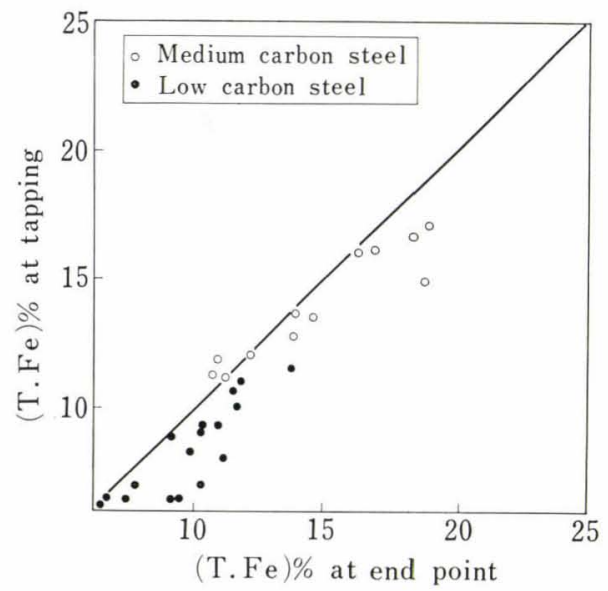

Fig. 7. Relation between T.Fe $\%$ at end point and at tapping for medium and high carbon steels

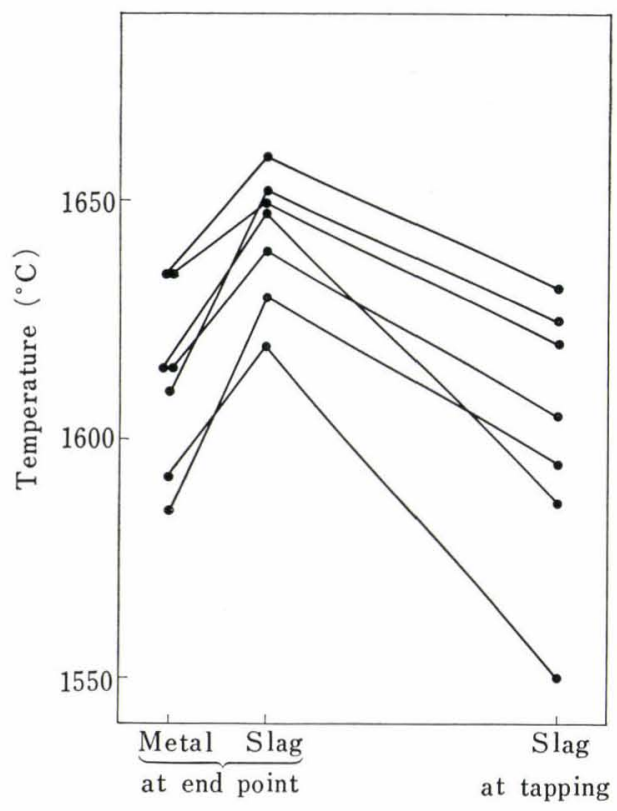

Fig. 8. Decrease of slag temperature after end point

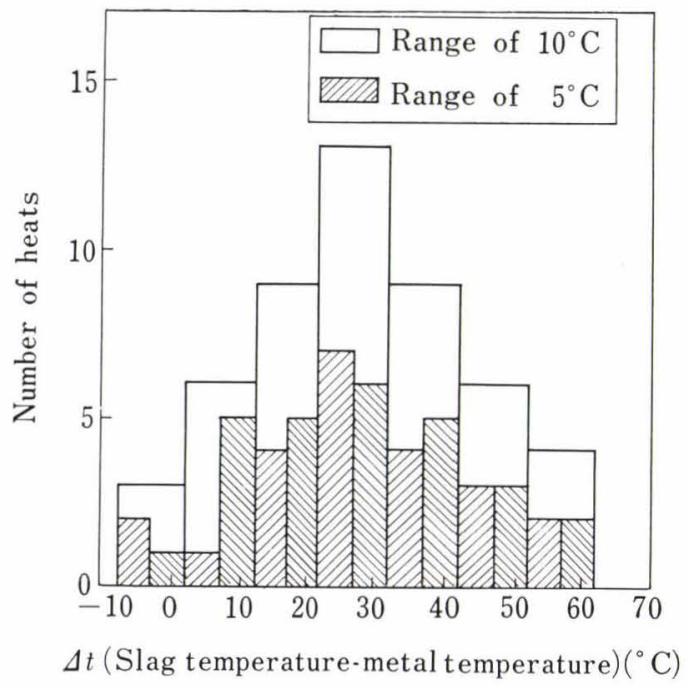

Fig. 9. The difference of temperature between metal and slag at end point
As may be seen in the figure, the dispersion of the data shows a regular distribution with the average temperature difference of $30^{\circ} \mathrm{C}$.

With regard to Fig. 9, the following must be taken into consideration: the time elapses from the oxygen blowing-down to the end of tilting and measurement of temperature is not constant, but is subjected greatly to the state of foaming of the slag. In the case of extensive foaming, it takes several minutes before the sedatives added calm down the slag. Therefore, the time from the oxygen blowing-down to the measurement of temperature varies widely from slightly over ten seconds to several minutes; long time intervals will allow the slag temperature to drop greatly from that at the oxygen blowing-down.

As a result of these investigations, it may be concluded that the difference of temperature between the slag and the metal is about $30^{\circ} \mathrm{C}$ on an average at the time when the furnace is tilted; probably it is over $50^{\circ} \mathrm{C}$ at the time of oxygen blowing-down.

\section{Analysis and Discussion}

1. On the Factor Affecting the Change of Oxygen in Metal in the Furnace for Low Carbon Steels

At first, the large change of oxygen for low carbon steels of Figs. 2 and 3 is analyzed, and the factor that affects it is sought. The result is shown in Fig. 10: it will be seen that the T.FeO of slag at the end point has a large effect upon the change of oxygen after the end point. This T.FeO is calculated by the following equation :

$$
\text { T. } \mathrm{FeO} \%=\mathrm{FeO} \%+1.35 \mathrm{Fe}_{2} \mathrm{O}_{3} \%
$$

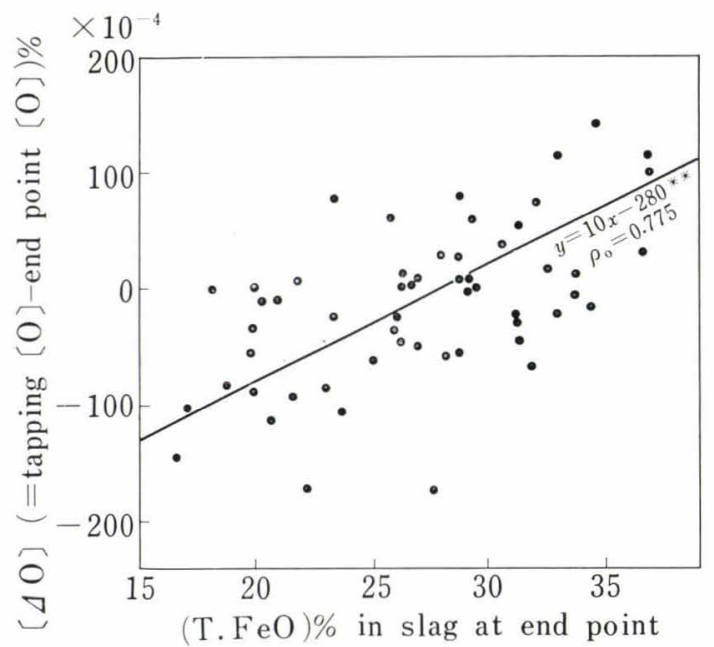

Fig. 10. The effect of T.FeO at end point on the change of oxygen after end point (End carbon $0.04 \leq \mathrm{C} \leq$ $0.08 \%$ )

The conventional result ${ }^{1,2)}$ is that the T.Fe in slag is not related to the oxygen in metal, not as it is in B.O.H. On the contrary, Fig. 10 shows that the iron oxide content at the end point is closely related to the change of oxygen content in metal after the end point. 
These two contradictory results may be understood as follows: during the blowing, and even at the blowing-down of oxygen, the metal and the slag are in a state of non-equilibrium; it takes several minutes of stay in the furnace for them to acquire the state of equilibrium, becoming to have a relation to each other.

Further, when T.FeO of the slag is high, the oxygen in the metal increases, whereas when the T.FeO is low, it decreases. Although it can be imagined easily that oxygen transfers itself from slag to metal for the former case, it is hard to see how oxygen does not escape as $\mathrm{CO}$ gas out of the furnace but merely moves from metal to slag in the latter. However, as it is unlikely that the oxygen can escape as CO gas out of the furnace only when the T.Fe is low, it must be that the oxygen does not escape as $\mathrm{CO}$ gas but moves into the slag.

\section{Calculation of the Degree of Oxidation of the Slag}

The equi-activity curve of $\mathrm{FeO}$ by Winkler and Chipman $^{3)}$ is shown in Fig. 11. The actual data $(0.04 \% \leq \mathrm{C}$ at the end point $\leq 0.08 \%)$ of 160 -t B.O.F.'s are plotted in this figure as quasi-ternary-system. The activity of FeO in slag is 0.15 to 0.40 . Substituting this activity into the following equation, oxygen equilibrated with slag can be calculated.

Assuming that Fig. 11 is applicable in the range of $1550^{\circ}$ to $1650^{\circ} \mathrm{C}$ :

$$
[\mathrm{O}]_{\text {cal. }} \%=a_{\mathrm{FeO}} \times[\mathrm{O}]_{\mathrm{equ} .} \%
$$

where, $[\mathrm{O}]_{\text {equ. }} \%$ : the soluble oxygen content that is equilibrated with $100 \% \mathrm{FeO}$ in pure iron and is a function of temperature.

The data of Chipman and Fetters and Taylor and Chipman $^{4}$ are applied to $[\mathrm{O}]_{\text {equ. } \%}$ here. If the temperature of slag is equal to that of metal, it is possible to compare $[\mathrm{O}]_{\text {cal. }} \%$ with the observed oxygen content of metal. Therefore, it is possible to compare the degrees of oxidation of slag and metal.

3. Comparison of the Degrees of Oxidation of Slag and Metal for Low Carbon Steels after the End Point

The degree of oxidation of slag is calculated by

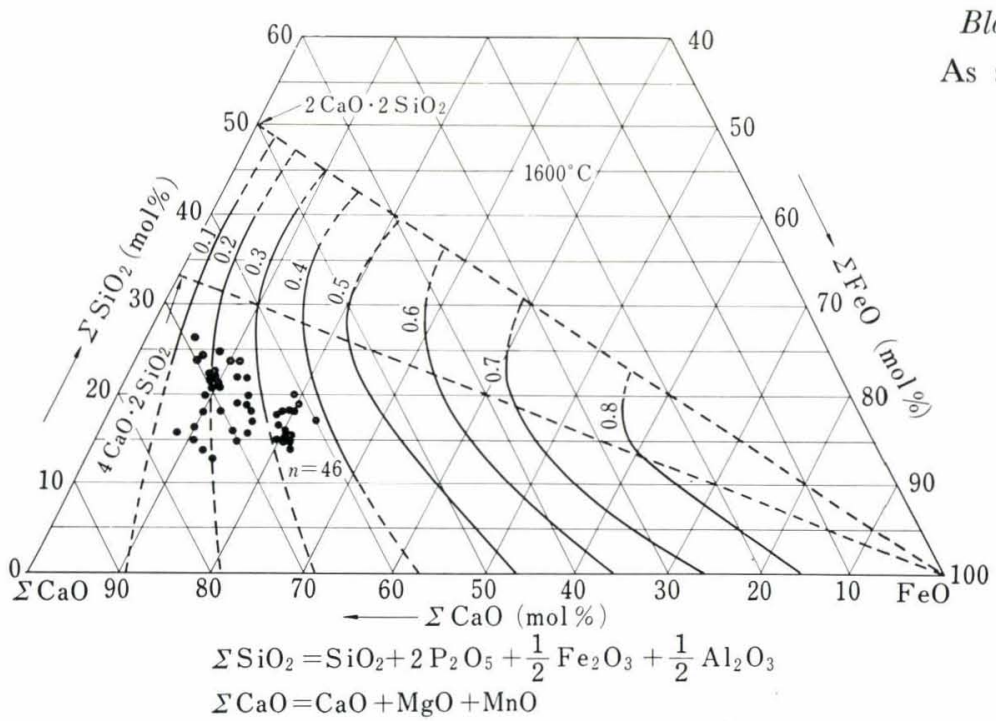

Fig. 11.

Activity of ferrous oxide in molten slag3)
Eq. (2). Now, taking the temperature of slag at the tapping to be equal to that of metal at the end point, the value by Eq. (2) gives the degree of oxidation of slag at the tapping according to Fig. 8. As mentioned above, the degree of oxidation of slag ([O] $]_{\text {cal. }} \%$ ) can be expressed in terms of the oxygen content of metal at equilibrium with slag.

The relation between the calculated oxygen content of slag $\left([\mathrm{O}]_{\text {cal. }} \%\right.$ ) and the observed oxygen content of metal $([\mathrm{O}] \%)$ at the end point or at the tapping is shown in Fig. 12. According to this result, approximately in one third of the heats investigated the oxygen content of metal is lower than that of slag. When the temperature of slag drops to the temperature of metal during the period from the end point to the tapping, the oxygen in metal moves to slag and its content decreases. In this figure, as the degree of scatter of the data at the tapping is not smaller than that at the end point, it may be concluded that the slag is not in the state of equilibrium with the metal even at the tapping.

Figure 13 shows the effect of the difference between actual oxygen ([O] end point) in metal and that calculated $\left([\mathrm{O}]_{\text {cal. }}\right.$ ) from slag composition upon the change in the oxygen content after the end point. In this figure, $[\Delta \mathrm{O}]_{\mathrm{cal}}$. is the difference in the degree of oxidation between slag and metal at the end point. Therefore, when the temperature of slag decreases to that of metal, $[\Delta \mathrm{O}]_{\mathrm{cal}}$, influences the change of oxygen in metal. That is, when the degree of oxidation of slag at the tapping is lower than that of metal, the oxygen in metal at the tapping becomes less than that at the end point, that much oxygen corresponding to this difference moving to slag. Conversely, when the degree of oxidation of slag is higher than that of metal, the oxygen in metal increases; in this case, oxygen should move from slag to metal. In the figure, however, it will be seen that the oxygen does not increase very much when $[\Delta \mathrm{O}]_{\text {cal }}$, is extremely high. This suggests that the oxygen transferred from slag to metal reacts with solute elements and cannot remain in metal.

4. Comparison of the Degrees of Oxidation of Slag and Metal during the Blowing and at the End Point (The Blowing-down)

As shown in Fig. 12, the degree of oxidation of 


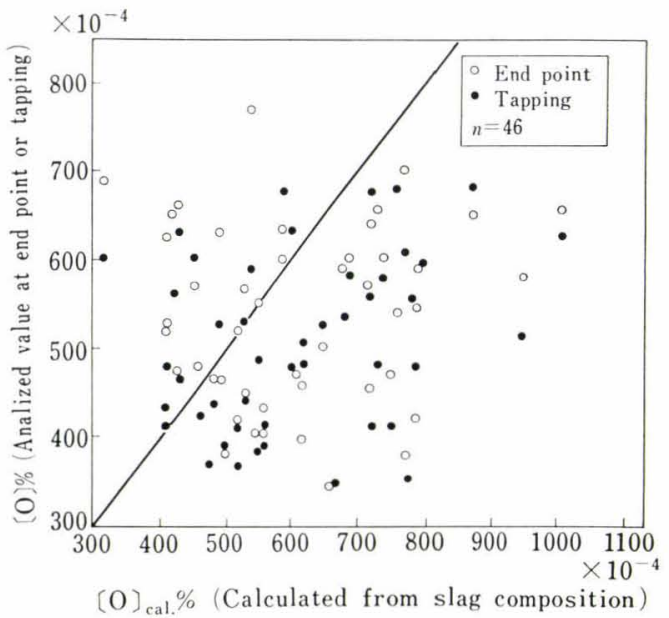

Fig. 12. Relation between $[\mathrm{O}]_{\mathrm{eal}}$. and $[\mathrm{O}]$ at end point or tapping (End point carbon $0.04 \leq \mathrm{G} \leq 0.08 \%$ )

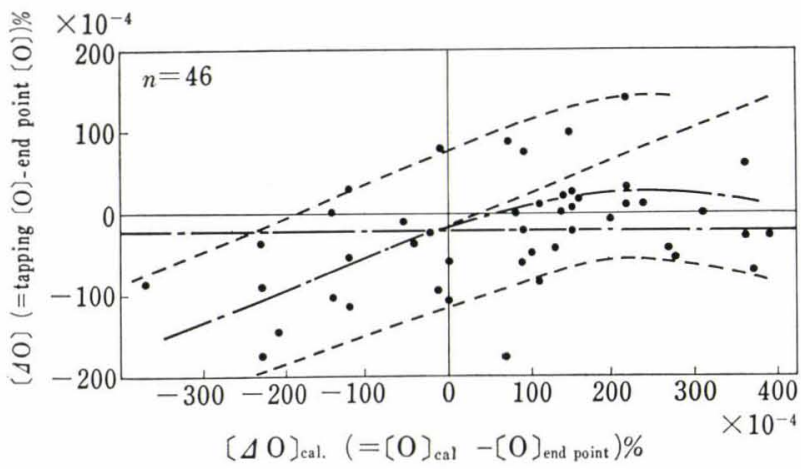

Fig. 13. The effect of the difference between actual oxygen in metal and that calculated from slag composition on the change in oxygen content after end point (End point carbon $0.04 \leq \mathrm{C} \leq 0.08 \%$ )

slag can become occasionally lower than that of metal when the temperature of slag drops to that of metal. During the blowing period, however, it has been shown earlier that the temperature of slag can be higher by over $50^{\circ} \mathrm{C}$ than that of metal. Therefore, it appears that this period need be investigated further.

At first, the difference of the degrees of oxidation of slag at $1600^{\circ}$ and $1650^{\circ} \mathrm{C}$ is calculated by Eq. (2), assuming that the temperature of slag is equal to that of metal and the composition of slag is unchanged. It is assumed further that the activity of $\mathrm{FeO}$ is 0.3 both at $1600^{\circ}$ and $1650^{\circ} \mathrm{C}$. The result of the calculation is :

$$
\begin{aligned}
& {[\mathrm{O}]_{\text {cal. }}^{1650^{\circ} \mathrm{C}}=0.084 \%} \\
& {[\mathrm{O}]_{\text {cal. }}^{1600^{\circ} \mathrm{C}}=0.069 \% .}
\end{aligned}
$$

If, however, the temperature of slag is not equal to that of metal, say, for example, the temperature of slag is $1650^{\circ} \mathrm{C}$ and that of metal is $1600^{\circ} \mathrm{C}$, a correction is necessary for the above result.

The difference of the oxygen contents in a metal with $0.06 \% \mathrm{C}$ is approximately $0.002 \%$ between $1600^{\circ}$ and $1650^{\circ} \mathrm{C}$. Applying this correction to the above result, the difference in the degrees of oxidation between slag and metal at $1650^{\circ} \mathrm{C}$ is higher by some $0.013 \%$ than that at $1600^{\circ} \mathrm{C}$.

Fitting this rough estimate to Fig. 12, it can be said on the whole that the degrees of oxidation of slag under the blowing and at the blowing-down are at least equal and probably higher than that of metal.

\section{On the Relation between $\mathrm{FeO}$ in Slag and Oxygen in Metal for Medium or High Carbon Steels}

For steels with over $0.10 \% \mathrm{C}$, i.e., medium or high carbon steels, the change of oxygen in metal in the furnace is small and the average of the change is slightly negative, as already shown in Fig. 3.

The degree of oxidation of slag, as calculated for such cases on the range of slag (T.Fe) from of Fig. 1 is shown in Fig. 14. Figure 14 includes two cases, that is, one is the case in which the temperature difference between slag and metal is $50^{\circ} \mathrm{C}$, and the other the case in which it is nil. According to the result, it may be concluded that for steels containing over $0.1 \% \mathrm{C}$, the difference in the degree of oxidation between slag and metal is the larger, the higher the carbon in metal. It may be seen from Fig. 14, further that the oxygen can no longer move from metal to slag, though it can move from slag to metal.

Figure 2 shows that the oxygen in the metal actually decreases slightly. This phenomenon is presumed to be a result of the following. Oxygen in metal together with oxygen moving from slag have been consumed in the decarburization reaction. Figure 5 supports this presumption. In the range of the higher carbon content decarburization of some $0.01 \% \mathrm{C}$ occurs on an average, for which oxygen of $0.0133 \%$ should have been consumed. Therefore, considering the decrease of oxygen in furnace, it is concluded that oxygen of over about $0.01 \%$ moves from slag to metal.

After all, for medium and high carbon steels, as the degree of oxidation of slag is usually higher than that

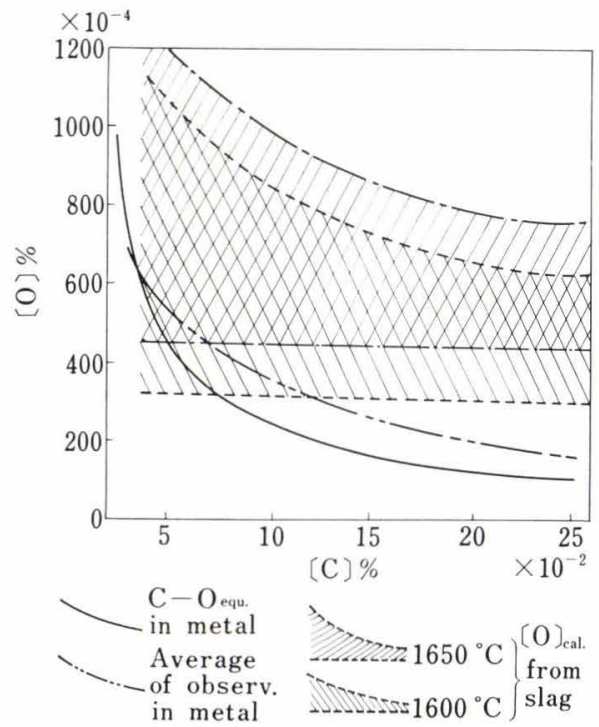

Fig. 14. Comparison of actual oxygen with oxygen calculated from slag composition 
of metal, oxygen can move from slag to metal virtually in one way only; almost all the oxygen moving from slag is consumed in the decarburization reaction, the situation that is markedly different from the cases of low carbon steels. Therefore, the oxygen content in metal does not apparently change at all.

\section{Summary and Conclusion}

The difference of temperature between slag and metal is not large regardless the carbon content of the steel. Therefore, the temperature of slag is higher by $50^{\circ} \mathrm{C}$ for the all carbon range than that of metal under the blowing and at the blowing-down of oxygen. This difference in temperature disappears during the several minutes from the oxygen blowing-down to the tapping.

In the blowing period and at the blowing-down, when there is the temperature difference of $50^{\circ} \mathrm{C}$, the degree of oxidation of slag is equal or higher than that of metal. When the difference of temperature disappears, however, the degree of oxidation of slag can become lower than that of metal, particularly for low carbon steels $(0.04 \% \leq \mathrm{C}$ at the end point $\leq 0.08 \%)$. In this case, oxygen in metal moves to slag, and the oxygen content decreases. Conversely, in the case that the degree of oxidation of slag is higher than that of metal, oxygen in slag moves to metal, where the oxygen content in metal becomes higher because the carbon content is low. Therefore, during the period from the end point to the tapping, oxygen in metal in the furnace increases or decreases according to the variation of the degree of oxidation of slag.

On the other hand, for medium and high carbon steels, even if the temperature of slag becomes equal to that of metal, the degree of oxidation of slag does not become lower than that of metal, and oxygen moves from slag to metal virtually in one way only. Moreover, as the oxygen which moves from slag into metal is consumed in the decarburization reaction, the oxygen content in metal does not apparently increase, a special characteristic of the medium and high carbon steels.

Although any relation between T.Fe in slag and the oxygen content in metal has not been detected at the end point in B.O.F., it has been observed frequently in operation that weakly deoxidized products are related with the heats of high T.Fe content. This apparent inconsistency has been shown to be the result of the variable relation between the T.Fe in slag and the oxygen in metal in that the equilibrium between slag and metal in the furnace takes several minutes to establish after the end point, and that the oxygen is transferred back and forth between slag and metal during this period according to the difference in temperature between slag and metal and to the variation of T.Fe of the slag.

Thus, it can be stated that in oxygen converter practice for low carbon steels, the control of the T.Fe in slag, which has a great influence on the oxygen content of steel, is very important.

\section{Acknowledgements}

The authors wish to thank Dr. Y. Doi, Manager of the Wakayama Steel Works of Sumitomo Metal Industries, Ltd., and Mr. M. Akabane, Assistant Manager of the same Works, for permission to publish the results. Thanks are also due to Mr. M. Toyoda for his assistant in this work.

\section{REFERENCES}

1) T. Fujii, T. Araki, and K. Marukawa: Tetsu-to-Hagané, 54 (1968), 153.

2) S. Tamamoto, T. Ikeda, and K. Marukawa: Tetsu-to-Hagané, 54 (1968), 385.

3) T. B. Winkler and J. Chipman: Trans. AIME, 167 (1946), 111.

4) J. Chipman: Basic Open Hearth Steelmaking, (1951), 628, AIME. 